\title{
Characterization of broadband complex refractive index of synthetic melanin coatings and their changes after ultraviolet irradiation $\odot$ (C)
}

Cite as: Appl. Phys. Lett. 117, 203701 (2020); https://doi.org/10.1063/5.0024229

Submitted: 05 August 2020 . Accepted: 26 October 2020 . Published Online: 17 November 2020

(i) Weiyao Li, (D) Anvay Patil, (iD) Xuhao Zhou, (iD) Zhao Wang, (iD) Ming Xiao, (iD) Matthew D. Shawkey, (iD) Nathan C. Gianneschi, and (iD) Ali Dhinojwala

\section{COLLECTIONS}

F This paper was selected as Featured

SCI This paper was selected as Scilight
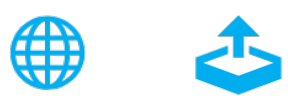

\section{ARTICLES YOU MAY BE INTERESTED IN}

Determining the refractive index of shocked [100] lithium fluoride to the limit of transmissibility

Journal of Applied Physics 116, 033515 (2014); https://doi.org/10.1063/1.4890714

An overview of the extremal index

Chaos: An Interdisciplinary Journal of Nonlinear Science 29, 022101 (2019); https://

doi.org/10.1063/1.5079656

Monthly average daily global and diffuse solar radiation based on sunshine duration and clearness index for Brasov, Romania

Journal of Renewable and Sustainable Energy 6, 053106 (2014); https://

doi.org/10.1063/1.4896596

\section{Challenge us.}

What are your needs for periodic signal detection?

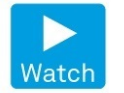

Zurich Instruments

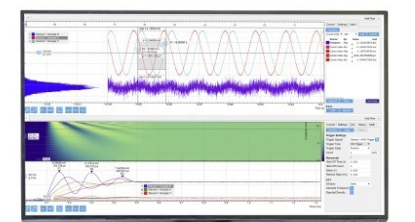

O 


\title{
Characterization of broadband complex refractive index of synthetic melanin coatings and their changes after ultraviolet irradiation $\odot$ ()
}

\author{
Cite as: Appl. Phys. Lett. 117, 203701 (2020); doi: 10.1063/5.0024229 \\ Submitted: 5 August 2020 • Accepted: 26 October 2020 . \\ Published Online: 17 November 2020
}
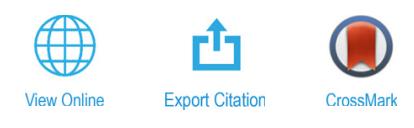

Weiyao Li, (D) Anvay Patil, ${ }^{1}$ (D) Xuhao Zhou, ${ }^{2}$ (D) Zhao Wang, ${ }^{2, a)}$ (D) Ming Xiao, ${ }^{1, b)}$ (D) Matthew D. Shawkey, ${ }^{3}$ Nathan C. Gianneschi, ${ }^{2,4, c)}$ (D) and Ali Dhinojwala, ${ }^{7, c)}$ (D)

\begin{abstract}
AFFILIATIONS
${ }^{7}$ School of Polymer Science and Polymer Engineering, The University of Akron, Akron, Ohio 44325, USA

${ }^{2}$ Department of Chemistry, International Institute of Nanotechnology, Simpson-Querrey Institute, Chemistry of Life Processes Institute, Lurie Cancer Center, Northwestern University, Evanston, Illinois 60208, USA

${ }^{3}$ Evolution and Optics of Nanostructures Group, Department of Biology, Ghent University, Ledeganckstraat 35, Chent 9000, Belgium

${ }^{4}$ Department of Materials Science and Engineering, Department of Biomedical Engineering, Department of Pharmacology, Northwestern University, Evanston, Illinois 60208, USA

${ }^{a)}$ Present address: Institute of Molecular Engineering, University of Chicago, Chicago, Illinois 60637, USA.

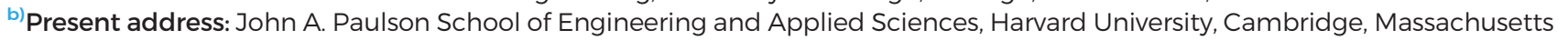
02138, USA.

${ }^{c}$ Authors to whom correspondence should be addressed: ali4@uakron.edu and nathan.gianneschi@northwestern.edu
\end{abstract}

\begin{abstract}
Melanin, with its high refractive index (RI) and broadband absorption, is an important biomaterial responsible for many of the vibrant structural colors observed in nature and for UV protection. Even though the RI plays an important role in the function of melanin, there is an ambiguity in its reported complex RI and a lack of understanding of whether and how the UV radiation, these materials are likely to experience under normal use, will affect the complex RI. Here, we measured the wavelength-dependent (360-1700 nm) complex RI of synthetic melanin films before and after in situ UV treatment using ellipsometry. We modeled the ellipsometric data using a modified TaucLorentz dispersion model and measured the thickness independently using atomic force microscopy. The UV radiation reduces the film thickness. Interestingly, we find that both the real and imaginary terms of the RI increase upon UV radiation. These experiments provide accurate measurements of the optical properties of melanin and a surprising result that synthetic melanin absorbs more light $(\sim 25 \%$ increase in extinction coefficient) below $600 \mathrm{~nm}$ after UV exposure.
\end{abstract}

Published under license by AIP Publishing. https://doi.org/10.1063/5.0024229

Melanin, a ubiquitous dark pigment widespread in many living organisms, is well known for protection against ultraviolet (UV) radiation. ${ }^{1-4}$ Melanin is described as a complex heterogeneous biopolymer and is not composed of one unique chemistry but instead represents black pigments found in various organisms including bacteria, fungi, plants, and animals. Currently, melanin is classified using five main groups that include eumelanin, pheomelanin, neuromelanin, allomelanin, and pyomelanin. ${ }^{1}$ All these different types of melanins are synthesized using different starting monomers. ${ }^{5-8}$ Eumelanin is one of the most studied melanins and consists of two main monomers, 5,6dihydroxyindole (DHI) and 5,6-dihydroxyindole-2-carboxylic acid
(DHICA), generated by oxidation of dopamine and L-3,4dihydroxyphenylalanine (L-DOPA). ${ }^{9}$ Synthetic melanin has been synthesized using dopamine and L-DOPA in the form of spherical particles with well-defined sizes and also coatings for membranes and surface modifications. ${ }^{10-13}$ Interestingly, the final polymerized form of melanin is also very heterogeneous and is held together by covalent bonds and secondary interactions (e.g., H-bonding and $\pi-\pi$ stacking), ${ }^{14}$ which complicates the structural elucidation with advanced characterization techniques such as solid-state nuclear magnetic resonance, infrared, and Raman spectroscopy. ${ }^{15-17}$ 
Nonetheless, this enigmatic material exhibits multifunctional properties, which attract interest from diverse disciplines. ${ }^{18}$ In particular, two unique optical properties of melanin have been of interest for decades. One is the high refractive index (RI, $\sim 1.7-1.8$ ), which results in vibrant structural colors ${ }^{11,19-27}$ and applications for medical diagnostics. ${ }^{28-30}$ The second is the broadband absorption, which is responsible for enhancing saturation in structural colors, ${ }^{11,19-27}$ protection against UV radiation, ${ }^{31,32}$ thermoregulation and imaging, ${ }^{33-35}$ and optical contrast in photoacoustic imaging. ${ }^{36}$ Both of the above properties are expected to be influenced by UV radiation, especially considering melanin's tendency to undergo structural changes when it acts as a UV-protective agent with UV-dissipative and radical-scavenging abilities. ${ }^{3,37}$ Recently, it has been shown that after high dosage of UV radiation, melanin synthesized using polydopamine (PDA) and L-DOPA degrades to release $\mathrm{CO}_{2}$, and some of the indole units are converted to a furopyrrole structure. ${ }^{17}$ Hence, there is a need to understand the optical properties of melanin and how it is affected after UV exposure.

Even though the complex RI $\left(n^{*}=n-i \kappa ; n\right.$ - real part, $\kappa$ - imaginary part) of melanin plays a vital role in structural colors and photoprotection, the quantification of $n^{*}$ for melanin has been challenging. The first report on the measurement of $n$ of melanin used a solvent contrast-matching method and estimated RI to be greater than 1.77 . $^{38}$ The wavelength dependence of $n$ for melanin in jewel beetles was reported to be from 1.79 to $1.64(400-700 \mathrm{~nm}) .^{39}$ Stavenga et al. derived the RI for melanin in tissues using an interference microscopy technique. $^{40,41}$ The wavelength dependence of $\kappa$ was measured by Xiao et al. using UV-vis absorption of synthetic melanin particles suspended in water. ${ }^{11}$ By using a low concentration of melanin, they minimized the scattering effect. ${ }^{42}$ However, scattering is not eliminated by this approach. Two recent studies used ellipsometry to measure $n^{*}$ for polyindoledione and PDA, which are synthetic melanins. ${ }^{36,43}$ However, the polyindoledione system lacks the chemical heterogeneity of typical melanin ${ }^{44}$ and the analysis on PDA did not yield Kramers-Kronig consistent values, which are required for highabsorbing materials, and underpredicted $n$ of melanin $(\sim 1.49)$.
Considering the importance of optical properties of melanin and the ambiguity of the reported RI values, there is a need to measure $n^{*}$ over a broad range of wavelengths.

Here, we report measurements of wavelength-dependent $n^{*}$ using ellipsometry from 360 to $1700 \mathrm{~nm}$ for melanin thin films prepared using two chemistries: PDA and poly(dopamine L-DOPA) (PDD). PDA is the most common form of synthetic melanin chemistry, and PDD chemistry is closer to that of natural melanin because of L-DOPA that is critical to the biosynthetic pathway. ${ }^{5}$ The melanin films were prepared on a silicon wafer through the one-step auto-oxidization process at the air-water interface of the melanin-polymerizing solution (Fig. 1), following a modified film-forming procedure (for experimental details, refer to the supplementary material). ${ }^{45}$ Recently, there have been suggestions that intermolecular assembly in melanin could lead to stacked structures along the growth direction. ${ }^{14}$ However, we provide evidence that including the uniaxial anisotropic model does not reduce the residuals. Therefore, we consider our system to be isotropic. Two steps were taken to implement a reliable analysis of the ellipsometer data. First, we directly measured the film thickness and roughness using atomic force microscopy (AFM). The directly measured thickness eliminated the "thickness" term as a fitting parameter in modeling ellipsometric parameters (delta $(\Delta)$ and psi $(\Psi))$. Second, since melanin is an amorphous high-absorbing material with structural complexity, ${ }^{15}$ we used the Kramers-Kronig consistent multioscillator Tauc-Lorentz (TL) dispersion model to calculate $n^{*}$ dispersion. This method was also used to determine how the optical properties of melanin change upon UV irradiation (320-450 nm, up to $2.36 \times 10^{8} \mathrm{~J} / \mathrm{g}$ ), a property that is critical for photoprotection. Our observations are consistent for the two chemistries in that both $n$ and $\kappa$ increase after radiation. These results suggest that the UV-protective function of melanin is enhanced after UV exposure.

We coated synthetic melanin on part of a silicon wafer for accurate measurement of the film thickness [Fig. 1(a); the film preparation methodology is described in the supplementary material]. Scanning
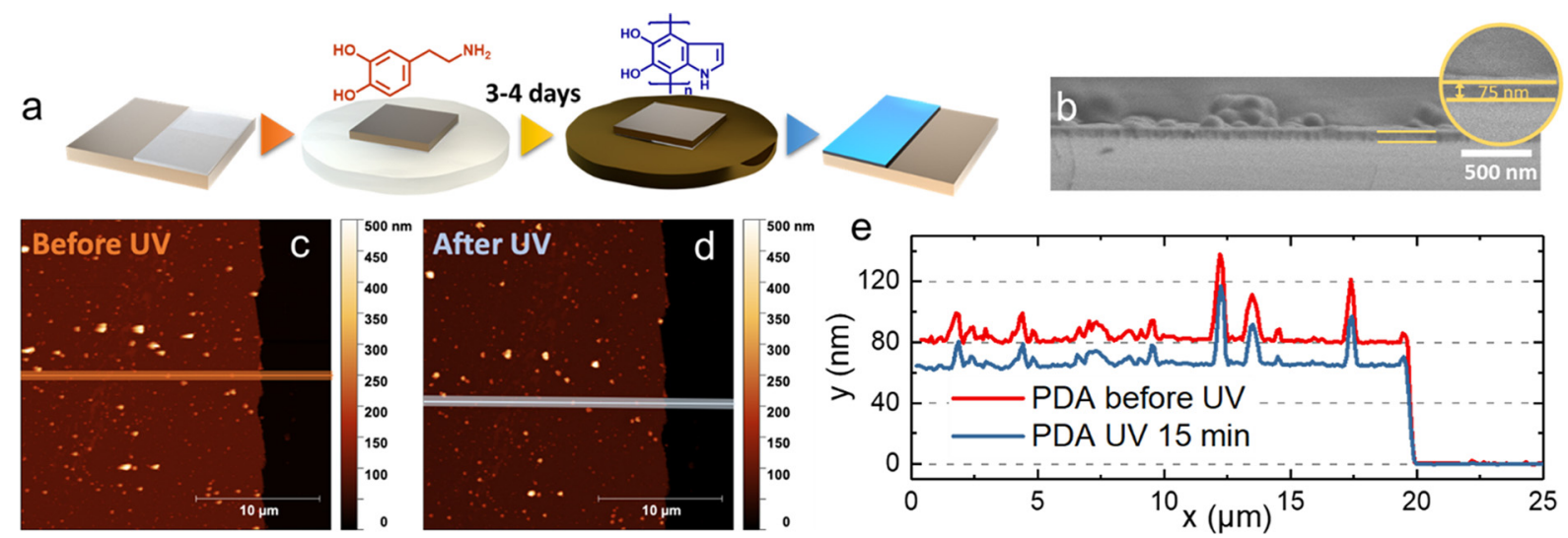

FIG. 1. (a) A diagram of the partial-coating process of synthetic melanin on the silicon wafer. A silicon wafer, partially covered with parafilm, is placed facing down on top of the melanin-polymerizing solution surface. After 3-4 days of reaction, a melanin film forms on the silicon wafer, resulting in a sample appearing blue. After the coating process, the parafilm is removed and the melanin-half-coated wafer is ultrasonicated in water for three times each to remove big particle residues. (b) SEM image of the cross section of the PDA film on the silicon wafer. (c) and (d) 2D AFM height sensor scans of the edge of a partially coated PDA film (c) before and (d) after UV treatment. (e) A line profile plot obtained by averaging ten-line scans [highlighted by the shaded regions in (c) and (d)] of the same region of the half-coated PDA film before and after UV treatment. 
electron microscopy (SEM) images of the film cross section reveal the presence of an aggregate of melanin residues on top of a uniform melanin coating, contributing to its roughness [Fig. 1(b)]. To quantify film thicknesses and thickness changes upon UV irradiation, the partially coated samples were measured using AFM to generate 2D height scans. For direct comparison, the height scans were performed at the same position for samples before [Figs. 1(c) and S2(a)] and after UV irradiation [Figs. 1(d) and S2(b)]. A representative dataset of PDA chemistry shows that the continuous layer thickness changes from $\sim 80 \mathrm{~nm}$ to $\sim 62 \mathrm{~nm}$ upon UV exposure [Fig. 1(e)], comparable to changes observed for UV-exposed polymer thin films. ${ }^{46-48}$ Similar results were also observed for the PDD chemistry [Fig. S2(c)]. The measured film thicknesses (continuous layer + roughness layer) are summarized in Table S1 for the PDA and PDD samples. We ensured that the ellipsometric parameters $(\Delta$ and $\Psi)$ of the synthetic melanin coatings on silicon wafers were acquired at the same spot as the AFM measurements before and after in situ UV treatment [Figs. 2(b) and (S6)] using a spectroscopic imaging ellipsometer (360-1700 nm).

The ellipsometric parameters, $\Delta(\lambda)$ and $\Psi(\lambda)$ in Figs. 2(b) and S6, are fit with a five-layer model, which consists of (a) polycrystalline silicon as a substrate, (b) a native oxide layer (silica or $\mathrm{SiO}_{2}$ ), (c) a continuous synthetic melanin coating layer, (d) a roughness layer, and (e) air as the ambient layer [Fig. 2(a)]. The roughness layer is modeled using a single-layer Bruggeman effective medium approximation (EMA), which follows a host-guest complex and can be expressed as $^{49}$

$$
\frac{\varepsilon_{\text {host }}-\varepsilon}{\varepsilon_{\text {host }}+2 \varepsilon}(1-f)+\frac{\left(\varepsilon_{\text {guest }}-\varepsilon\right)}{\left(\varepsilon_{\text {guest }}+2 \varepsilon\right)} f=0
$$

where $f$ is the fraction of the guest (air) component, $\varepsilon_{\text {host }}$ is the dielectric dispersion of the host (melanin component), which will be discussed below, $\varepsilon_{\text {guest }}$ is the dielectric dispersion of the guest (air) component, and $\varepsilon$ is the dielectric dispersion of the effective medium. Typically for thin films with high surface roughness, advanced methods involving a gradient EMA model are used for modeling roughness. ${ }^{50}$ However, the gradient EMA model gave us similar results to those obtained using single-layer EMA (Fig. S7). We have also tried other models for roughness (Lorentz-Lorentz and Maxwell-Garnett EMA) and not seen any improvements in the fitting residuals. The wavelength-by-wavelength analysis of $\Delta(\lambda)$ and $\Psi(\lambda)$ (Fig. S8), by solving Fresnel equations for the multi-layer thin film model, exhibits unexpected shapes of $n^{*}$ of synthetic melanin for both before and after UV irradiation. The $n$ presents a bimodal peak in the visible region before UV irradiation while a prominent single peak after UV irradiation. For both the treatment groups, we observe a broad extinction tail in $\kappa$. These observations guided us to develop a Kramers-Kronig consistent complex dielectric dispersion $\left(\varepsilon^{*}=\varepsilon_{1}\right.$ $-i \varepsilon_{2} ; \varepsilon_{1}$-real part and $\varepsilon_{2}$-imaginary part) for synthetic melanin using a multi-oscillator TL model. This modified TL dispersion contains a Lorentz oscillator, a Gaussian oscillator (only used for before UV treatment), and a pole-UV oscillator to account for dispersion created by absorption outside the measured spectral range in the UV region. The imaginary part of the dielectric constant $\left(\varepsilon_{2}\right)$ of TL dispersion is given by ${ }^{51}$

$$
\varepsilon_{2}(E)=G_{T}(E) L(E),
$$

a
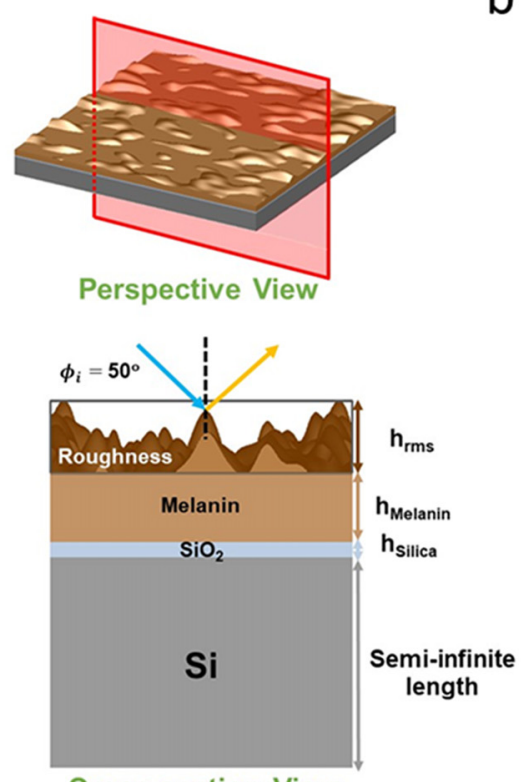

Cross-section View b
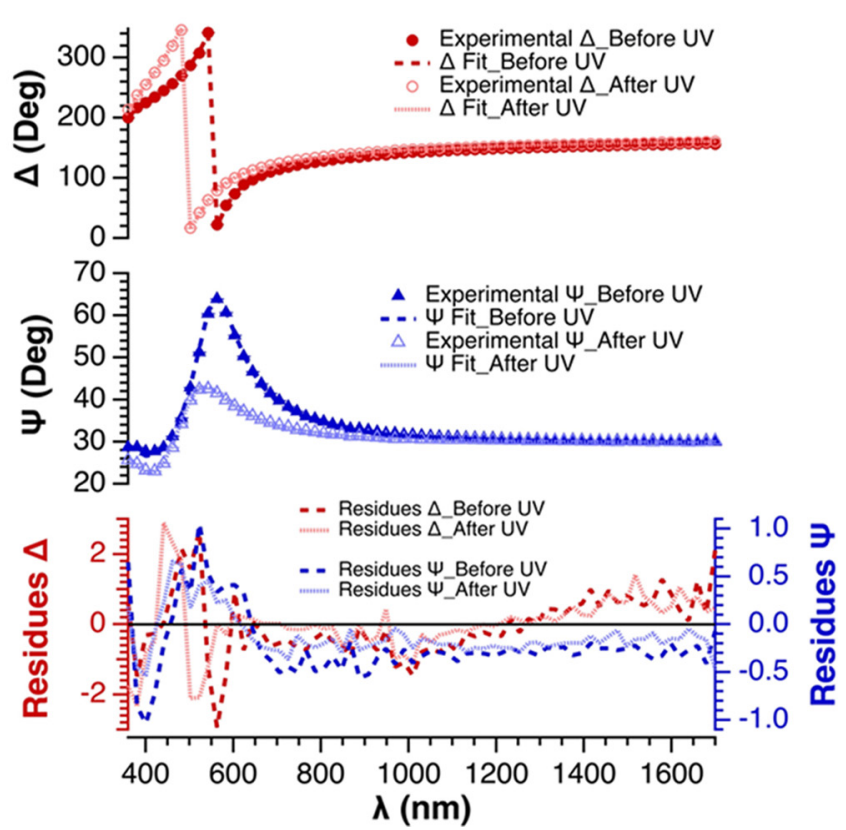

FIG. 2. (a) A CAD schematic of the optical model used for the synthetic melanin thin film in the modeling of $\Delta$ and $\Psi$. (b) $\Delta$ and $\Psi$ curves of PDA measured by the spectroscopic ellipsometer before (filled circles and triangles, respectively) and after in situ UV treatment (hollow circles and triangles, respectively) along with their fitting curves (dashed lines) modeled using the multi-term dispersion [Eq. (6)]. A lower residual of $\Delta$ and $\Psi$ indicates a better fit. 


$$
G_{T}(E)= \begin{cases}\frac{A_{T}\left(E-E_{g}\right)^{2}}{E^{2}}, & E>E_{g} \\ 0, & E \leq E_{g}\end{cases}
$$

and it is called the Tauc gap function ${ }^{52}$ ( $A_{T}$ is the Tauc amplitude), indicating interband mechanisms above the band edge and, thus, no absorption when photon energy $(E)$ is lower than bandgap energy $\left(E_{g}\right)$, and $L(E)$ is given by

$$
L(E)=\frac{A_{L} E_{0 L} \Gamma E}{\left(E^{2}-E_{0 L}^{2}\right)^{2}+\Gamma^{2} E^{2}},
$$

and it is the imaginary part of the complex dielectric function for a single classical Lorentz oscillator ${ }^{53}\left(A_{L}\right.$ is the oscillator amplitude, $E_{O L}$ is the energy (position) of the Lorentz peak, and $\Gamma$ is the broadening parameter). Equation 2(c) is also used to express the imaginary part $\left(\varepsilon_{2}\right)$ of an independent Lorentz oscillator term. The Gauss oscillator produces a Gaussian line shape, and its parameterized form of the imaginary part $\left(\varepsilon_{2}\right)$ is given by

$$
\varepsilon_{2}(E)=A_{G}\left(e^{\frac{-\left(E-E_{c}\right)^{2}}{\sigma^{2}}}-e^{\frac{-\left(E+E_{c}\right)^{2}}{\sigma^{2}}}\right),
$$

where

$$
\sigma=\frac{B}{2 \sqrt{\ln 2}}
$$

Here, $A_{G}$ is the absorption amplitude, $E_{c}$ is the oscillator energy, and $B$ is the bandwidth of resonance. The real part of dielectric functions $\left(\varepsilon_{1}\right)$ is calculated using the Kramers-Kronig integration of $\varepsilon_{2}$ functions for all oscillator types [Eqs. (2), (2b), and (3)] and is given by ${ }^{53}$

$$
\varepsilon_{1}(E)=\varepsilon_{\infty}+\frac{2}{\pi} P \int_{0}^{\infty} \frac{\xi \varepsilon_{2}(\xi)}{\xi^{2}-E^{2}} d \xi,
$$

where $\varepsilon_{\infty}$ is the value of the real part of the dielectric function at infinite energy and is used as an additional parameter in the multioscillator TL model. The value of $\varepsilon_{\infty}$ is fixed at 1 . The $P$ in Eq. (4) stands for the Cauchy principal part of the integral. $\xi$ is the energy variable with respect to which the integration is performed. Additionally, the pole-UV oscillator is equivalent to a Lorentz oscillator with zerobroadening. It affects only the real part of the dielectric function $\left(\varepsilon_{1}\right)$,

$$
\varepsilon_{1}(E)=\frac{A_{P}}{E_{0 P}^{2}-E^{2}},
$$

where $A_{P}$ is the pole amplitude and $E_{O P}$ is the pole central energy and $\varepsilon_{2}$ is equal to zero. It is important to note that all the energy parameters are in units of electronvolts $(\mathrm{eV})$. Thus, the final expression for multiterm dielectric dispersion (Fig. S9) is written as

$$
\varepsilon(E)=\varepsilon_{\infty}+\varepsilon_{\text {Tauc-Lorentz }}+\varepsilon_{\text {Lorentz }}\left(+\varepsilon_{\text {Gauss }}\right)+\varepsilon_{\text {pole_UV }} .
$$

With independently measured thicknesses of the melanin films on corresponding spots by AFM [Figs. 1(c) and 1(d)], we fit the $\Delta(\lambda)$ and $\Psi(\lambda)$ curves by adjusting the fitting parameters in the multi-term model (listed in Table S2) including the fraction of air in the roughness layer. After observing small changes in some of the parameters $\left(E_{O P}\right.$, $A_{L}, E_{O L}$, and $\Gamma$ ) during the fitting of the multivariate expression, we decide to fix those parameters to reduce fitting uncertainties. When isolating the individual contribution of each term to the overall multiterm dielectric dispersion (Fig. S9), the TL term dominates the general shape of dielectric distribution and provides a bandgap to determine the primary absorption region, disregarding any defect-related or intraband absorptions. The Lorentz term helps expand the tail of absorption to lower energy, where it cannot be typically described by a TL model for amorphous material, implicating the presence of secondary structures. In the case of before-UV treatment, the TL term alone cannot capture the optical dispersion behavior as one isotropic, amorphous component. This observation suggests a second population of absorptive structures [whose contribution to the absorption peak lies closer to the UV region (2.5-3.4 eV)], which could be effectively represented by a Gaussian distribution (typical of amorphous semiconductors) $)^{55}$ and, thus, a Gauss oscillator term is incorporated. Interestingly, after UV irradiation, the two resolvable contributions merge into a single, amorphous distribution and the Gauss oscillator term is no longer needed. It is important to note that the sum of all the oscillator terms does not fully describe the $\varepsilon_{1}$ component of multi-term dielectric dispersion. Hence, a single pole-UV term is introduced that increases $\varepsilon_{1}$ throughout the whole wavelength region to match the data. Since the complex dielectric dispersion of melanin is unlike other semiconducting materials, which has an absorption maximum, ${ }^{56}$ the combination of the above oscillators turns out to be a good representation for the melanin system. Furthermore, to test the robustness of the proposed multi-term dielectric dispersion, we fit $\Delta(\lambda)$ and $\Psi(\lambda)$ curves measured at varying angles of incidences with the same model to yield lower residuals of $\Delta$ and $\Psi$ (indicative of good fit) (Fig. S10). To test the effect of anisotropy on the optical constants along the direction normal to the film surface, we also fit our ellipsometric data using the same multi-oscillator dielectric dispersion with a uniaxial anisotropic model. Compared to the isotropic model presented earlier, the application of the uniaxial anisotropic model neither improves the residuals of $\Delta$ and $\Psi$ (Fig. S11) nor shows significant differences between the planar and perpendicular dielectric constants.

The relationship between complex dielectric dispersion and $n^{*}$ can be given as ${ }^{53}$

$$
\begin{gathered}
\varepsilon_{1}(E)=n^{2}(E)-\kappa^{2}(E), \\
\varepsilon_{2}(E)=2 n(E) \kappa(E),
\end{gathered}
$$

which can be further solved to obtain $n^{*}$ from complex dielectric constants,

$$
\begin{aligned}
& n(\lambda)=\left[\frac{\sqrt{\varepsilon_{1}^{2}(\lambda)+\varepsilon_{2}^{2}(\lambda)}+\varepsilon_{1}(\lambda)}{2}\right]^{1 / 2}, \\
& \kappa(\lambda)=\left[\frac{\sqrt{\varepsilon_{1}^{2}(\lambda)+\varepsilon_{2}^{2}(\lambda)}-\varepsilon_{1}(\lambda)}{2}\right]^{1 / 2},
\end{aligned}
$$

where

$$
\lambda(\mathrm{nm})=\frac{1239.84}{E(\mathrm{eV})}
$$

The distribution of optical constants for the two synthetic melanin chemistries and two treatment groups as a function of wavelength are shown in Fig. 3. Both PDA and PDD exhibit a peak $(\sim 500 \mathrm{~nm})$ in $n$ with the highest RI values reaching $1.81 \pm 0.01$ and $1.86 \pm 0.05$, respectively, for before UV treatment and $1.88 \pm 0.01$ and $1.94 \pm 0.06$ 

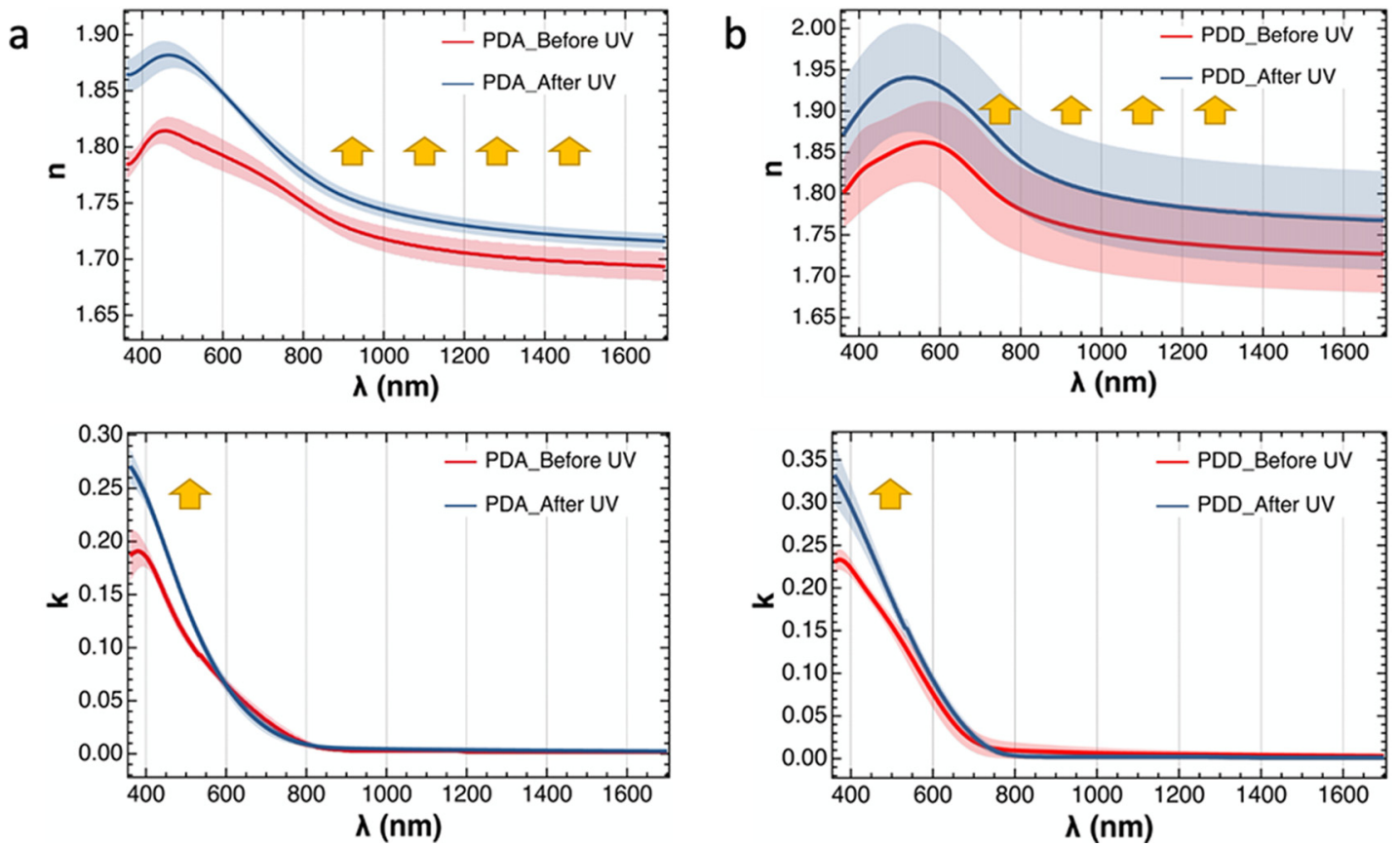

FIG. 3. (a) Real $(n)$ and imaginary ( $\kappa$ ) parts of the complex RI $\left(n^{*}\right)$ of the PDA film before (red curve) and after (blue curve) in situ UV treatment. (b) $n$ and $\kappa$ of the PDD film before (red curve) and after (blue curve) in situ UV treatment. The error ranges (shown by the shaded regions) are the standard deviations calculated from optical constants, which are obtained from calculations with the parameters in Table S2 for each chemistry.

a
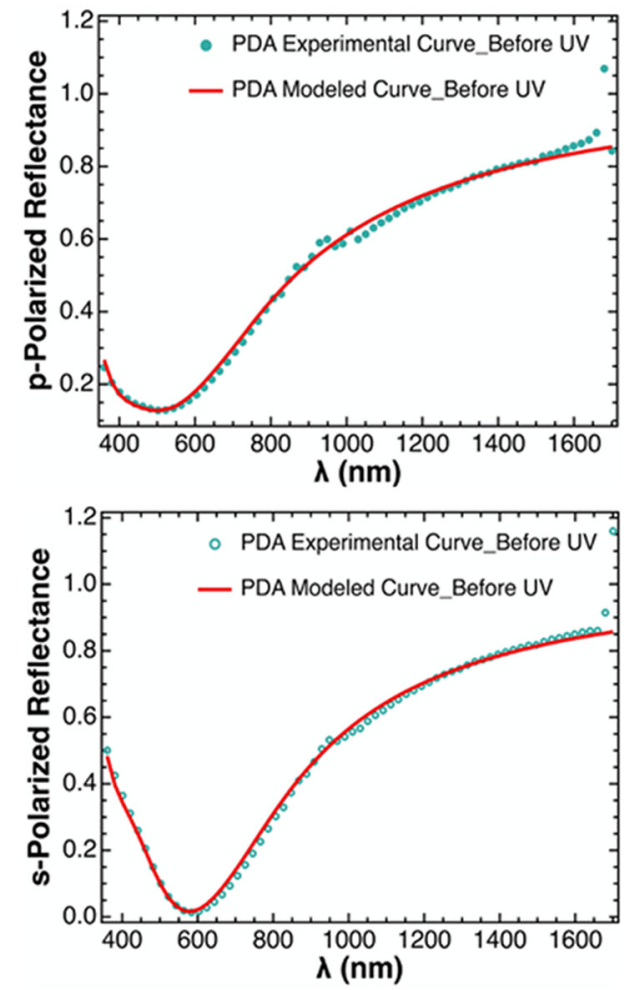

b
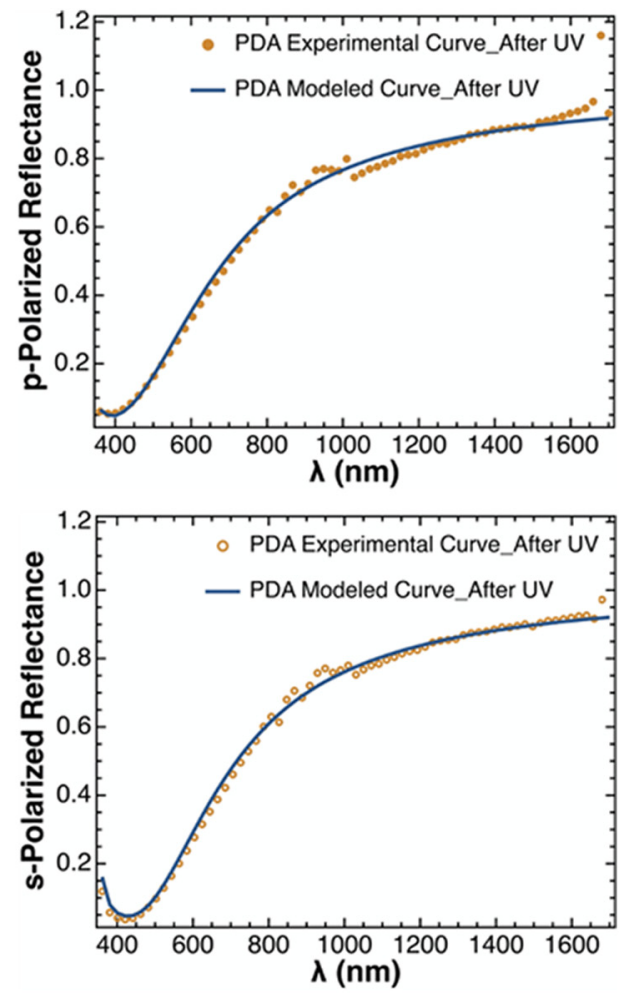

FIG. 4. p-(solid circles) and s-polarized (hollow circles) reflectance spectra of the PDA film (a) before (cyan) and (b) after (yellow) in situ UV treatment along with their modeled curves (lines; red for before and blue for after UV) generated using a multi-layer interference model with the obtained $n^{*}$ values of PDA for both the cases. 
for after UV treatment. In general, the values of $n$ (over a range of $360-1700 \mathrm{~nm}$ ) and $\kappa$ (below $600 \mathrm{~nm}$ ) of both PDA and PDD significantly increased after UV exposure $\left(n: \mathrm{F}_{1,10}=36.7, \mathrm{P}=0.0001 ; \kappa\right.$ : $\mathrm{F}_{1,10}=67.7, \mathrm{P}<0.0001$; for details on statistical methods, refer to the supplementary material). Moreover, PDD exhibits higher $n$ and $\kappa$ than PDA $\left(n: \mathrm{F}_{1,10}=33.6, \mathrm{P}=0.0002 ; \kappa: \mathrm{F}_{1,10}=126.7, \mathrm{P}<0.0001\right)$, likely due to the presence of the polymerized form of L-DOPA in PDD in addition to PDA.

After UV exposure, the film thicknesses (continuous layer + roughness layer) decrease by $18.5 \pm 5.9 \%$ for PDA and $13.8 \pm 4.5 \%$ for PDD. Moreover, the treatment results in an increase (up to $4.5 \%$ ) in $n$ from 360 to $1700 \mathrm{~nm}$ and an $\sim 25 \%$ increase in the $\kappa$ component for both chemistries in regions below $600 \mathrm{~nm}(\sim 36 \%$ especially $<400 \mathrm{~nm}$ ). The increase in the $\kappa$ component is particularly interesting because it suggests that melanin becomes a more efficient UV blocker after UV exposure. From a molecular perspective, the chemical structure of melanin particles partially changes from the indole to furopyrrole structure with the release of carbon dioxide after UV irradiation. ${ }^{17}$ We anticipate that similar changes take place for melanin films as well. The similarity in FTIR spectra between particles and films, for both before and after UV irradiation (Fig. S12), indicates that the results we have obtained for PDA and PDD films could be similar for synthetic melanin particles prepared using similar chemistry. The solid state nuclear magnetic resonance spectroscopy results indicate that there is an increase in the aromatic content and a decrease in the aliphatic content upon UV exposure, ${ }^{17}$ which partially explains the increase in $n$ and $\kappa .^{57,58}$ Further research involving density functional calculations to establish the structure-property relationship is necessary to validate this hypothesis.

To evaluate the reliability of our calculated Kramers-Kronig consistent $n^{*}$, we measured the reflectance spectra of melanin films (PDA chemistry as a representative system) at both p- and s-polarized incident light $(360-1700 \mathrm{~nm})$ and fit them with theoretical reflectance curves generated using a multi-layer interference model with the obtained $n^{*}$ values of PDA for both the treatment groups. The excellent agreement between the experimental and modeled reflectance curves demonstrates the accuracy of RI measurements performed in this study (Fig. 4).

In summary, we have measured the $n^{*}$ for synthetic melanin films before and after UV treatment. The deconstruction of components in the multi-term dielectric dispersion provide insights into relating the changes in optical properties to the changes in the chemical structure of melanin after UV irradiation. Surprisingly, melanin absorbs more light $(<600 \mathrm{~nm})$ after intense UV exposure, which could be a mechanism to explain melanin's photoprotective behavior. Furthermore, an improved measurement of wavelength-dependent $n^{*}$ paves the way for the design of melanin-based materials for optical applications.

See the supplementary material for the details of experimental methods, selected data plots of PDD samples, fitting parameters for the multi-oscillator model, and other supporting figures and tables to the main manuscript.

\section{AUTHORS' CONTRIBUTIONS}

A.P. and W.L. contributed equally to this work. W.L., A.P., and A.D. contributed to project conceptualization and design of experiments. A.P., W.L., and X.Z. performed the experiments. A.P. and W.L. analyzed the data. Z.W. and M.X. provided guidance during the experimentation phase. W.L. and A.P. drafted the first version, and this manuscript was finalized after contributions from all the authors. All authors have given approval to the final version of this manuscript.

The authors thank Cheng Liu and Steven S.C. Chuang for the use of their FTIR equipment and Abraham Joy for his UV spot lamp. A.D., A.P., and W.L. thank the National Polymer Innovation Center (NPIC) and Andrew Knoll for providing access and technical assistance on the ellipsometer. A.P. and W.L. would also like to thank Matthias Duwe and Arash Mirhamed (Accurion $\mathrm{GmbH}$ ) for their input on modeling ellipsometer data. The authors would also like to thank Austin Garner for his assistance in statistical analysis. We also extend our gratitude to Mario Echeverri and Saranshu Singla for helpful discussion and comments on this manuscript. The authors acknowledge financial support from the Air Force Office of Scientific Research (AFOSR) under the MultiUniversity Research Initiative (MURI) Grant (No. FA9550-181-0142). M.D.S. acknowledges support from an AFOSR Grant (No. FA9550-18-1-0477), an FWO Grant (No. G007117N), and an HFSP Grant (No. RGP 0047).

\section{DATA AVAILABILITY}

The data that support the findings of this study are available within the article and its supplementary material.

\section{REFERENCES}

'L. D'Alba and M. D. Shawkey, Physiol. Rev. 99, 1 (2019).

${ }^{2}$ M. Brenner and V. J. Hearing, Photochem. Photobiol. 84, 539 (2008).

${ }^{3}$ A. B. Mostert, S. B. Rienecker, C. Noble, G. R. Hanson, and P. Meridith, Sci. Adv. 4, eaaq1293 (2018).

${ }^{4}$ D. N. Peles and J. D. Simon, J. Phys. Chem. B 115, 12624 (2011).

${ }^{5}$ J. D. Simon and D. N. Peles, Acc. Chem. Res. 43, 1452 (2010).

${ }^{6}$ M. d'Ischia, A. Napolitano, V. Ball, C. Chen, and M. J. Buehler, Acc. Res. Chem. 47, 3541 (2014).

7J. D. Nosanchuk, R. E. Stark, and A. Casadevall, Front. Microbiol. 6, 1463 (2015).

${ }^{8}$ A. Vasanthakumar, A. Dearaujo, J. Mazurek, M. Schilling, and R. Mitchell, Microbiology 161, 1211 (2015).

${ }^{9} \mathrm{G}$. Prota, Melanins and Melanogenesis (Academic Press, Inc., San Diego, 1992).

${ }^{10}$ D. J. Kim, K. Ju, and J. Lee, Bull. Korean Chem. Soc. 33, 3788 (2012).

${ }^{11}$ M. Xiao, Y. Li, M. C. Allen, D. D. Deheyn, X. Yue, J. Zhao, N. C. Gianneschi, M.

D. Shawkey, and A. Dhinojwala, ACS Nano 9, 5454 (2015).

${ }^{12}$ F. Solano, Int. J. Mol. Sci. 18, 1561 (2017).

${ }^{13}$ J. Kuang, J. L. Guo, and P. B. Messersmith, Adv. Mater. Interfaces 1, 1400145 (2014).

${ }^{14}$ S. Hong, Y. Wang, S. Y. Park, and H. Lee, Sci. Adv. 4, eaat7457 (2018).

${ }^{15}$ J. Liebscher, R. Mrówczyński, H. A. Scheidt, C. Filip, N. D. Hădade, R. Turcu, A. Bende, and S. Beck, Langmuir 29, 10539 (2013).

${ }^{16}$ Z. Huang, H. Lui, X. K. Chen, A. Alajlan, D. I. McLean, and H. Zeng, J. Biomed. Opt. 9, 1198 (2004).

${ }^{17}$ W. Li, Z. Wang, M. Xiao, T. Miyoshi, X. Yang, Z. Hu, C. Liu, S. S. C. Chuang, M. D. Shawkey, N. C. Gianneschi, and A. Dhinojwala, Biomacromolecules 20, 4593 (2019).

${ }^{18}$ W. Xie, E. Pakdel, Y. Liang, Y. J. Kim, D. Liu, L. Sun, and X. Wang, Biomacromolecules 20, 4312 (2019).

${ }^{19}$ M. Xiao, A. Dhinojwala, and M. Shawkey, Opt. Express 22, 14625 (2014).

${ }^{20}$ M. Fan, L. D’Alba, M. D. Shawkey, A. Peters, and K. Delhey, Biol. J. Linn. Soc. 128, 550 (2019).

${ }^{21}$ J. Zi, X. Yu, Y. Li, X. Hu, C. Xu, X. Wang, X. Liu, and R. Fu, Proc. Natl. Acad. U. S. A. 100, 12576 (2003).

${ }^{22}$ M. D. Shawkey and G. E. Hill, J. Exp. Biol. 209, 1245 (2006). 
${ }^{23}$ A. Kawamura, M. Kohri, S. Yoshioka, T. Taniguchi, and K. Kishikawa, Langmuir 33, 3824 (2017).

${ }^{24}$ M. Echeverri, A. Patil, Z. Hu, M. D. Shawkey, N. C. Gianneschi, and A. Dhinojwala, ACS Appl. Mater. Interfaces 12, 19882 (2020).

${ }^{25}$ R. Maia, J. V. O. Caetano, S. N. Báo, and R. H. Macedo, J. R. Soc. Interface 6, S203 (2009).

${ }^{26}$ M. Xiao, Z. Hu, Z. Wang, Y. Li, A. D. Tormo, N. L. Thomas, B. Wang, N. C. Gianneschi, M. D. Shawkey, and A. Dhinojwala, Sci. Adv. 3, el701151 (2017).

${ }^{27}$ M. Echeverri, A. Patil, M. Xiao, W. Li, M. D. Shawkey, and A. Dhinojwala, ACS Appl. Mater. Interfaces 11, 21159 (2019).

${ }^{28}$ M. Rajadhyaksha, M. Grossman, D. Esterowitz, R. H. Webb, and R. R. Anderson, J. Invest. Dermatol. 104, 946 (1995).

${ }^{29}$ A. N. Bashkatov, E. A. Genina, V. I. Kochubey, M. M. Stolnitz, T. A. Bashkatova, O. V. Novikova, A. Y. Peshkova, and V. V. Tuchin, Proc. SPIE 4162, 219 (2000).

${ }^{30}$ L. K. Ferris and R. J. Harris, Dermatol. Clin. 30, 535 (2012).

${ }^{31}$ E. S. Krol and D. C. Liebler, Chem. Res. Toxicol. 11, 1434 (1998).

${ }^{32}$ Y. Huang, Y. Li, Z. Hu, X. Yue, M. T. Proetto, Y. Jones, and N. C. Gianneschi, ACS Cent. Sci. 3, 564 (2017).

${ }^{33}$ M. D. Shawkey, B. Igic, S. Rogalla, J. Goldenberg, S. Clusella-Trullas, and L. D’Alba, Sci. Nat. 104, 78 (2017).

${ }^{34}$ S. Rogalla, L. D'Alba, A. Verdoodt, and M. D. Shawkey, J. R. Soc. Interface 16, 20190032 (2019).

${ }^{35}$ Y. Zou, T. Wu, N. Li, X. Guo, and Y. Li, Polymer 186, 122042 (2020).

${ }^{36}$ T. Repenko, A. Rix, A. Nedilko, J. Rose, A. Hermann, R. Vinokur, S. Moli, R. Cao-Milàn, M. Mayer, G. von Plessen, A. Fery, L. De Laporte, W. Lederle, D. N. Chigrin, and A. J. C. Kuehne, Adv. Funct. Mater. 28, 1705607 (2018).

${ }^{37}$ A. Huijser, A. Pezzella, and V. Sundström, Phys. Chem. Chem. Phys. 13, 9119 (2011).
${ }^{38}$ W. J. Schmidt, Naturwissenschaften 39, 313 (1952).

${ }^{39}$ S. Yoshioka and S. Kinoshita, Phys. Rev. E 83, 051917 (2011).

${ }^{40}$ D. G. Stavenga, H. L. Leertouwer, and B. D. Wilts, Light Sci. Appl. 2, e100 (2013).

${ }^{41}$ D. G. Stavenga, H. L. Leertouwer, D. C. Osorio, and B. D. Wilts, Light Sci. Appl. 4, e243 (2015).

${ }^{42}$ G. H. Meeten, Meas. Sci. Technol. 8, 728 (1997).

${ }^{43}$ M. Vega, E. M. Martın del Valle, M. Perez, C. Pecharroman, and G. Marcelo, ChemPhysChem 19, 3418 (2018).

${ }^{44}$ T. G. Barclay, H. M. Hegab, S. R. Clarke, and M. Ginic-Markovic, Adv. Mater. Interfaces 4, 1601192 (2017).

${ }^{45}$ O. Y. Milyaeva, A. G. Bykov, R. A. Campbell, G. Loglio, R. Miller, and B. A. Noskov, Colloids Surf., A 579, 123637 (2019).

${ }^{46}$ Handbook of Thin Film Deposition, edited by K. Seshan (Elsevier Science, Netherlands, 2012).

${ }^{47}$ M. Rashidian and D. Dorranian, J. Theor. Appl. Phys. 8, 121 (2014).

${ }^{48}$ M. D. Migahed and H. M. Zidan, Curr. Appl. Phys. 6, 91 (2006).

${ }^{49}$ D. A. G. Bruggeman, Ann. Phys. 416, 636 (1935).

${ }^{50}$ D. Lehmann, F. Seidel, and D. R. T. Zahn, SpringerPlus 3, 82 (2014).

${ }^{51}$ G. E. Jellison and F. A. Modine, Appl. Phys. Lett. 69, 371 (1996).

${ }^{52}$ J. Tauc, R. Grigorovici, and A. Vanvu, Phys. Status Solidi 15, 627 (1966).

${ }^{53}$ H. Fujiwara, Spectroscopic Ellipsometry: Principles and Applications (Wiley, Germany, 2007).

${ }^{54}$ R. A. Synowicki and T. E. Tiwald, Thin Solid Films 455-456, 248 (2004).

${ }^{55}$ J. E. McGinness, Science 177, 896 (1972).

${ }^{56}$ C. M. Ramsdale and N. C. Greenham, J. Phys. D: Appl. Phys. 36, L29 (2003).

${ }^{57} \mathrm{~T}$. Higashihara and M. Ueda, Macromolecules 48, 1915 (2015).

${ }^{58}$ N. C. Billingham, in Encyclopedia of Polymer Science and Technology, 4th ed. (John Wiley \& Sons, Inc., 2002), p. 34. 